http://www.jfas.info

\title{
FPGA IMPLEMENTATION OF CORDIC ALGORITHM IN DIGITAL MODULATION
}

A. S. N. Mokhtar ${ }^{*}$, S. A. Karim, S. P. Chew, S. M. F. S. M. Dardin, L. S. Supian and F. R. Hashim

Department of Electric and Electronics, Faculty of Engineering, National Defence University of Malaysia, 57000 Kuala Lumpur, Malaysia

Published online: 10 September 2017

\begin{abstract}
The modern communication systems and software radio based applications' demands fully digital receivers, consisting of only an antenna and a fully programmable circuit with digital modulators and demodulators. A basic communication system's transmitter modulates the amplitude, phase or frequency proportional to the signal being transmitted. This paper involves implementation of CORDIC (Coordinate Rotation Digital Computer) algorithm for generating digital modulation Amplitude shift keying (ASK) and Phase Shift keying (PSK). CORDIC was introduced to implement and versatile algorithm widely used for digital signal processing application that works only with shifter and shifter. A digital modulation is presented, the result obtained on Modelsim-Altera are given to illustrate the efficiency and the benefits of the proposed approach and implemented in DE-115 FPGA for real time applications.
\end{abstract}

Keywords: ASK; PSK; CORDIC algorithm; FPGA.

Author Correspondence, e-mail: anis@upnm.edu.my

doi: http://dx.doi.org/10.4314/jfas.v9i3s.23 


\section{INTRODUCTION}

In the past few decades, thousands of technologies and inventions especially in the science and engineering field have been drastically developed. Signal processing and image processing, communications, modulations, robotics, graphics control, neural network and motor control drive are examples of the rapid technologies invention [1]. In electronics or broadcastings, modulation is the route of conveying a message signal inside another signal that can be physically transmitted. Digital modulation is the transmission of digitally modulated analogue signals (carriers) between two or more points in a communication system [2]. There are three key factors of a periodic waveform which is amplitude, phase and frequency. The main reason for modulation is relating to the laws of electromagnetics propagation and to allow simultaneous transmission of different signals.

In digital modulation, the modulation is applied in response to the digital information. Over the past few years a major transition has occurred from simple analogue Amplitude Modulation (AM) and Frequency/Phase Modulation (FM/PM) to new digital modulation techniques. Some of the example of basic digital modulation technique is Amplitude Shift keying (ASK), Frequency Shift Keying (FSK), Phase Shift Keying (PSK) and Quadratic Phase Shift Keying (QPSK) [3]. ASK is a linear and sensitive to atmospheric and distortion [2]. It can be divided into three blocks which are transmitter, channel and receiver.

The advantages of ASK is it reduce in the amount of energy required to transmit information and it required small bandwidth to modulate or demodulate signal compare to FSK. Phase shift keying (PSK) is a form of angle-modulated and constant amplitude digital modulation [2]. In PSK, the phase of the sinusoidal carrier is changed to indicate the information. There are several methods that can be used to accomplish PSK. The advantages of PSK is it allows data to be carried along a radio communications signal much more efficiently than with frequent shift keying [3-6].

\subsection{CORDIC Algorithm}

CORDIC stands for Coordinate Rotation Digital Computer. The key concept of CORDIC arithmetic is based on the simple and olden principles of two-dimensional geometry. Invention of CORDIC by Jack E. Volder has been recorded in history in 1959 [7-9]. His 
discovery is an explanation to the navigation computer problems at that time. As time passes, his invention has been studied and developed continuously by other scientists and mathematicians [7-8]. The beauty of CORDIC is that by a simple shift-add operations, it is proficient to perform several computing tasks such as calculation of trigonometric functions including sine, cosine, magnitude and phase, hyperbolic and logarithmic functions, real and complex multiplications, division, square root, solution of linear system, singular value decomposition and many other tasks.

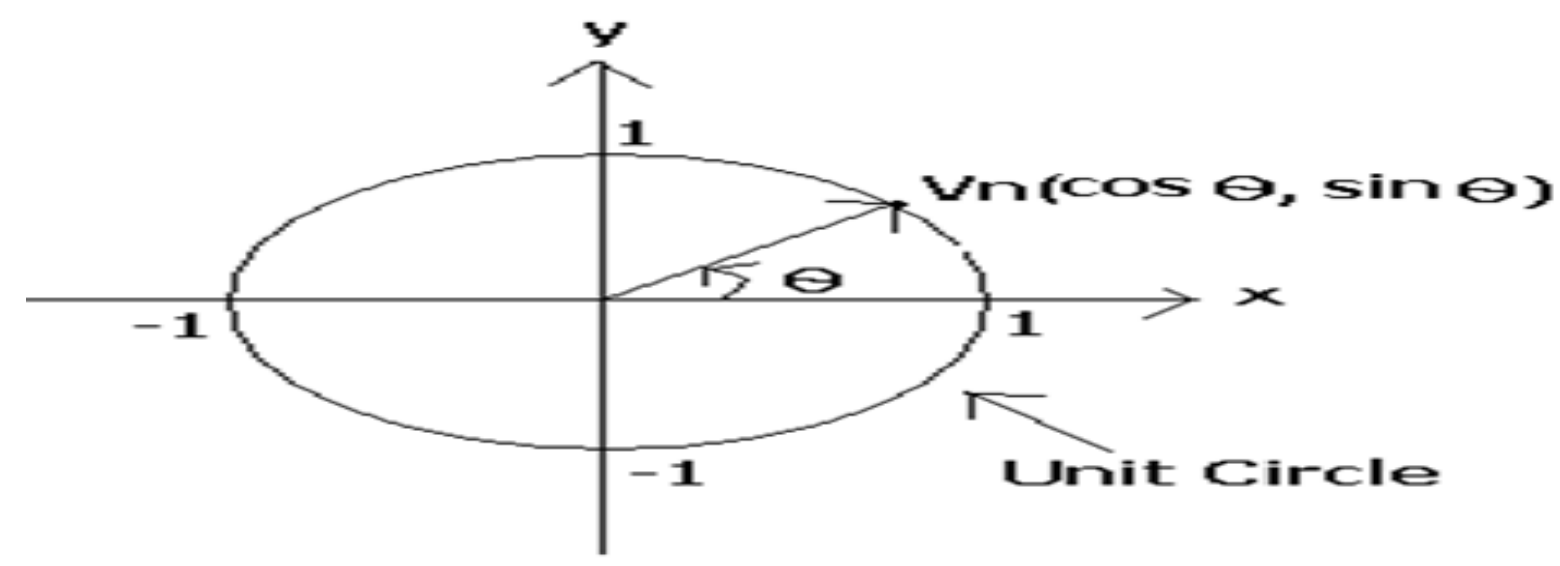

Fig.1. Unit cycle with vector, Vn

The CORDIC algorithm provides an iterative method in order to performing vector rotations by coordinate angles by using add and shift operations. Basically, CORDIC algorithm comprises of two working modes which is rotation mode and vectoring mode individually. The "rotation" described by Volder, which involved rotates the input vector by a specified. The second is called "vectoring", which only involved rotation of the input vector on the $x$-axis while recording the angle desired during that rotation [7-8]. According to the Fig. 1, the coordinate on the unit circle which has a rotation of $\theta$ has both $\cos \theta$ and $\operatorname{sine} \theta$ coordinates. This prove that if a point on the $x$-axis is rotated by an angle $\theta$, the cosine and sine of that angle of rotation would be read directly for both $x$ and $y$ axis. This vector rotation could be achieved by rotating the coordinate on the circle system in series steps, which are much smallerthan $\theta$. These steps would be either in a clockwise $(-\theta)$ or anti-clockwise $(+\theta)$. As an example, if we desired to achieve a total rotation of $45^{\circ}$, first we are rotating the vector $30^{\circ}$ in anti-clockwise, then rotate $20^{\circ}$ anti-clockwise direction and finally rotate the vector $5^{\circ}$ clockwise. 
Table 1. $\theta$ and $\tan \theta$ values

\begin{tabular}{ccc}
\hline Rotation, $\mathbf{N}$ & $\boldsymbol{\Theta}$ & Tan $\boldsymbol{\Theta}$ \\
\hline 0 & $45.000^{\circ}$ & $1=2^{0}$ \\
1 & $26.565^{\circ}$ & $\frac{1}{2}=2^{-1}$ \\
2 & $14.036^{\circ}$ & $\frac{1}{4}=2^{-2}$ \\
3 & $7.125^{\circ}$ & $\frac{1}{8}=2^{-3}$ \\
4 & $3.576^{\circ}$ & $\frac{1}{16}=2^{-4}$ \\
5 & $1.789^{\circ}$ & $\frac{1}{32}=2^{-5}$ \\
6 & $0.895^{\circ}$ & $\frac{1}{64}=2^{-6}$ \\
7 & $0.448^{\circ}$ & $\frac{1}{128}=2^{-7}$ \\
\hline
\end{tabular}

The coordinates of a point in 2-dimension space is indicate as a vector. If coordinates of the points are $(x, y)$, then the point would be indicate as $(x, y)^{\prime}$ where the inverted comma presents a matrix transpose function. This rotation of a point in two dimensional spaces basically because of multiplication of the coordinates of that point by a rotation matrix,

$$
\begin{gathered}
{\left[\begin{array}{cc}
\cos \theta & -\sin \theta \\
\sin \theta & \cos \theta
\end{array}\right]\left[\begin{array}{l}
x \\
y
\end{array}\right]} \\
=\left[\begin{array}{l}
x^{\prime} \\
y^{\prime}
\end{array}\right]
\end{gathered}
$$

So, the result is,

$$
\begin{aligned}
& x^{\prime}=x^{*} \cos \theta-y^{*} \sin \theta \\
& y^{\prime}=y^{*} \cos \theta+x^{*} \sin \theta
\end{aligned}
$$

Then both Equation (2) and (3) are divided with $\cos \theta$ :

$$
\begin{aligned}
& \frac{x^{\prime}}{\cos \theta}=x-y \tan \theta \\
& \frac{y^{\prime}}{\operatorname{sine} \theta} \\
& =x+y \tan \theta
\end{aligned}
$$

Based on to both equations above, $\theta$ are presents the rotation for each step. It is possible to 
separate the rotation into many steps, each of step is decreasing size such since $\tan \theta$ is the power of two. As a result, $\tan \theta$ can be implemented by the multiplication as a very efficient bit shift operation. Table 1 indicates the $\theta$ value along with $\tan \theta$ [7-8]. Since the rotation may be either in a counter-clockwise direction or clockwise, it is clearly that these steps would be used to approximate an angle between -99.4 and -99.4 $\left(45^{\circ}+\right.$ $+3.576^{\circ}+1.789^{\circ}+0.448^{\circ}+26.565^{\circ}+14.036^{\circ}+7.125+0.895=$ 99.434).

Furthermore, the decreasing size of steps presents that the approximation could be made arbitrarily accurate by increasing the number of rotation steps. Lookup table is used in order to keep the angle $\theta$ for every step. The table size increases with $\log _{2} N$ where $N$ indicates the number of step rotation. By referring to the Equation (4) and (5), $\tan \theta$ is needed in order to effect the rotation of a point along the unit circle. The CORDIC algorithm in every mode is limited between $-\pi / 2$ and $\pi / 2$. This limitation is caused by the first rotation angle $\theta_{o}=\tan ^{-1}\left(2^{-i}\right)$. The basic idea of CORDIC lies rotate $(0,1)$ by $\theta$ degree to get $x=x \cos \theta, y=y \sin \theta$ (Helbert and Meyr, 1999). Lookup table is used in order to keep the angle $\theta$ for every step. The table size increases with $\log _{2} N$, where $\mathrm{N}$ indicates the number of step rotation. By referring to the Equation (4) and (5), $\tan \theta$ is needed in order to effect the rotation of a point along the unit circle. By assuming the $\theta$ is between $90^{\circ}$ and $-90^{\circ}$, then the trigonometric identity yields

$\cos \theta=\cos (-\theta)$

This shows that if our rotational steps are always the similar angles, it makes no difference if our rotation steps are clockwise our anti-clockwise direction. This would be gives us to substitute a constant in place of the $\cos \theta$ in term of both Equation (4) and (5). The constant depends on the number of steps of the rotation to approximate the cosine and sine values:

$\cos \theta=\cos \left(\tan ^{-1} 2^{-i}\right)$

For example, if 4 rotation steps are used to approximate the sine and cosine values, then the constant $\mathrm{A}_{4}$ is calculated as follows:

$\mathrm{A}_{4}=\cos \left(\tan ^{-1} 1\right)+\cos \left(\tan ^{-1} \frac{1}{2}\right) \ldots+\cos \left(\tan ^{-1} \frac{1}{16}\right)+\cos \left(\tan ^{-1} \frac{1}{32}\right) \ldots$ 
So that, the iteration number can be represent as:

$x_{i+1}=A_{i}\left[x_{i}-y_{i} \cdot d_{i} \cdot 2^{-i}\right]$

$y_{i+1}=A_{i}\left[y_{i}-x_{i} \cdot d_{i} \cdot 2^{-i}\right]$

where

$$
A_{i}=\cos \left(\tan ^{-1} 2^{-i}\right)=1 / \sqrt{1+2^{-2 i}}, d_{i}= \pm 1
$$

The particular angle of a rotation can be distinctively defined by the sequence of the directions of the elementary rotations. To compute the angles desired, an additional adder-subtractor that accumulates the elementary angles for iterations is used:

$Z_{i+1}=Z_{i}-d_{i} *\left(2^{-i}\right)$

Thus, the CORDIC rotator's (using rotation mode) equations are:

$x_{i+1}+1=x_{i}-y_{i} * d_{i} \cdot 2^{-1}$

$y_{i+1}+1=y_{i}-x_{i} * d_{i} \cdot 2^{-1}$

$Z_{i+1}+1=Z_{i}-d_{i} * \tan ^{-1} \cdot 2^{-1}$

where

$d_{i}=-1$ if $Z_{i}<0,+1$ otherwise

By using CORDIC algorithm, the digital modulation technique can be implemented such as ASK, PSK, FSK and QPSK. Digital modulation is more secure and more efficient in long distance transmission. It has important place in modern communication because it required minimum number of blocks necessary for achieving the modulation. The input carrier signal and message signal are generated using CORDIC algorithm. These digital modulators were developed and compiled in ModelSim to generate waveform.

\subsection{Amplitude Shift Keying}

Amplitude-shift keying (ASK) is a form of amplitude modulation that represents digital data as variations in the amplitude of a carrier. In an ASK system, the binary symbol 1 is represented by transmitting a fixed-amplitude carrier wave and fixed frequency for a bit duration of $\mathrm{T}$ seconds [10]. If the signal value is 1 , then the carrier signal will be transmitted. Otherwise, a signal value of 0 will be transmitted. Fig. 1 illustrates a binary ASK signal (lower) together with binary sequence which initiated it (upper). Neither signal has been 
bandlimited. The expression of ASK is as shown in Equation (12) [11].

$s(t)= \begin{cases}A_{c} \cos \left(\omega_{c} t\right) & \text { Binary ' } 1 ' \\ 0 & \text { Binary ' } 0 \text { ' }\end{cases}$

where $A_{c}$ is the peak amplitude of the carrier signal and $\omega_{c}$ is the angular frequency of the carrier signal.

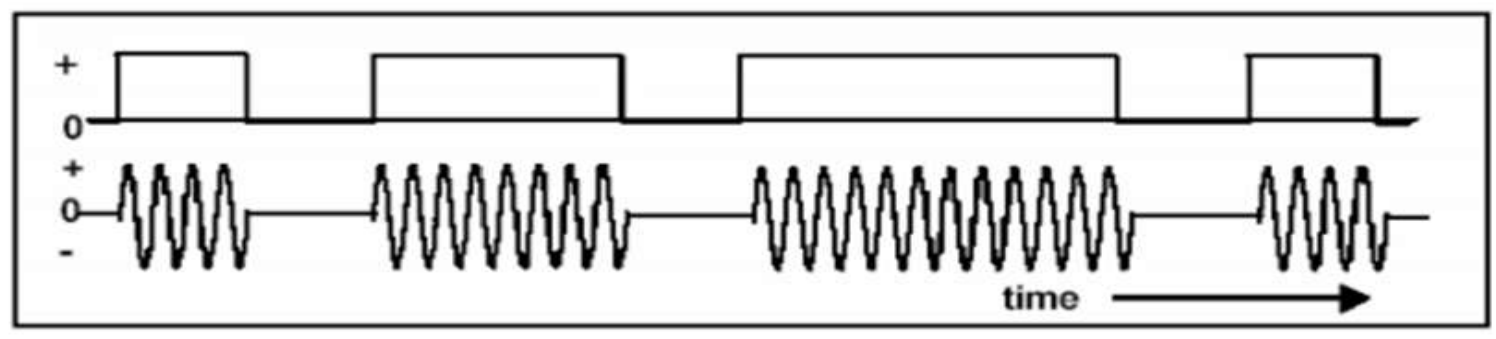

Fig.2.An ASK signal (below) and the message signal

\subsection{Phase Shift Keying (PSK)}

Phase Shift Keying (PSK) is a method of digital communication in which the phase of transmitted signal is varied to transmit information. Phase-shift keying (PSK) is a digital modulation scheme based on changing or modulating the initial phase of a carrier signal. The changing of phase is shown in

Fig.3.The changing of phasePSK is used to represent digital information such as binary digits 0 and 1 [4]. PSK is being used because it offers the lowest probability error. PSK is a form of angle-modulated and constant amplitude digital modulation. With PSK, the input is binary digital signal and there are a limited number of output phases possible. PSK modulation has proved to be a particularly suited for digital transmission over radio-relay links [12]. In PSK, the phase of the sinusoidal carrier is changed to indicate the information. The Equation of PSK is shown in Equation (12).

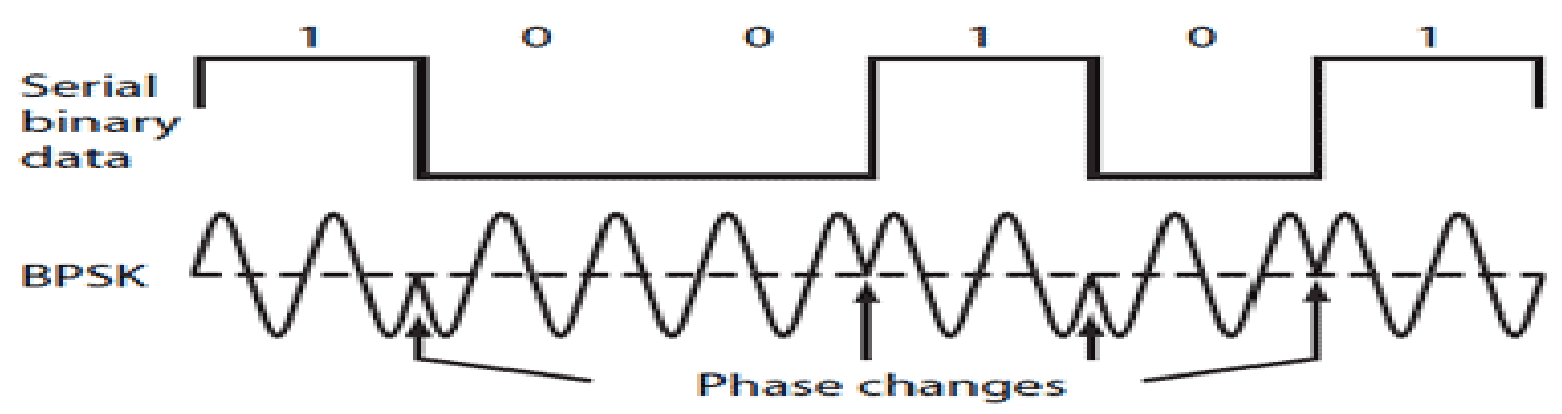

Fig.3.The changing of phase 


\section{METHODOLOGY}

Rotation mode of CORDIC algorithm is used in developing the digital modulation. The output of CORDIC algorithm is further processed to implement the ASK and PSK signal by applying Equation (4)-(7). The input theta for both schemes was determined as input $f$ the CORDIC algorithm. The rotation by an angle $\theta$ is implemented as an iterative process. From Equation (1)-(3), the pre-rotation of the angle was calculated until 10th iterations as example shown in Table 1 for $\theta=15^{\circ}$. The output $x_{i+1}$ shows the value for $\cos \theta$ while the value for $y_{i+1}$ shows the value of $\sin \theta$.

Table 2.Calculation of CODRIC algorithm for $15^{\circ}$

\begin{tabular}{ccccccc}
\hline $\mathbf{x}_{\mathbf{i}}$ & $\mathbf{i}$ & $\mathbf{2}^{\wedge}-\mathbf{i}$ & $\operatorname{Arctan}^{\wedge} \mathbf{- 1}$ & $\mathbf{x}_{\mathbf{i}+\mathbf{1}}$ & $\mathbf{y}_{\mathbf{i}+\mathbf{1}}$ & $\mathbf{Z}_{\mathbf{i}+\mathbf{1}}$ \\
\hline 1 & 0 & 1.0000 & 0.7853 & 0.6070 & 0.6070 & -0.5235 \\
2 & 1 & 0.5000 & 0.4636 & 0.9105 & 0.3035 & -0.0599 \\
3 & 2 & 0.2500 & 0.2449 & 0.9863 & 0.0758 & 0.1859 \\
4 & 3 & 0.1250 & 0.1234 & 0.9644 & 0.2602 & 0.0606 \\
5 & 4 & 0.0625 & 0.0624 & 0.9644 & 0.2602 & -0.0017 \\
6 & 5 & 0.0312 & 0.3112 & 0.9725 & 0.2300 & 0.0294 \\
7 & 6 & 0.0156 & 0.0156 & 0.9689 & 0.2452 & 0.0138 \\
8 & 7 & 0.0078 & 0.0078 & 0.9670 & 0.2528 & 0.0060 \\
9 & 8 & 0.0039 & 0.0039 & 0.9660 & 0.2566 & 0.0021 \\
10 & 9 & 0.0019 & 0.0019 & 0.9655 & 0.2585 & 0.0001 \\
11 & 10 & 0.0009 & 0.0009 & 0.9653 & 0.2594 & -0.0007 \\
12 & 11 & 0.0004 & 0.0004 & 0.9654 & 0.2589 & -0.0002 \\
13 & 12 & 0.0002 & 0.0002 & 0.9655 & 0.2586 & $-4.542 \mathrm{E}$ \\
14 & 13 & 0.0001 & 0.0001 & 0.9655 & 0.2586 & $7.664 \mathrm{E}$ \\
\hline
\end{tabular}

The output at 10th iteration is taken since it gives the most accurate result as the calculated value. Fig. 3 shows the architecture of digital modulation by interrogating CORDIC as medium. The input of the system consists of the input $\theta$, which connected directly to the CORDIC's module and the amplitude of the system. The output of the CORDIC's module 
denote as $\mathrm{x}_{\mathrm{i}+1}$ is further used to complete the modulation and the modulated signal are obtained at the end of the result.

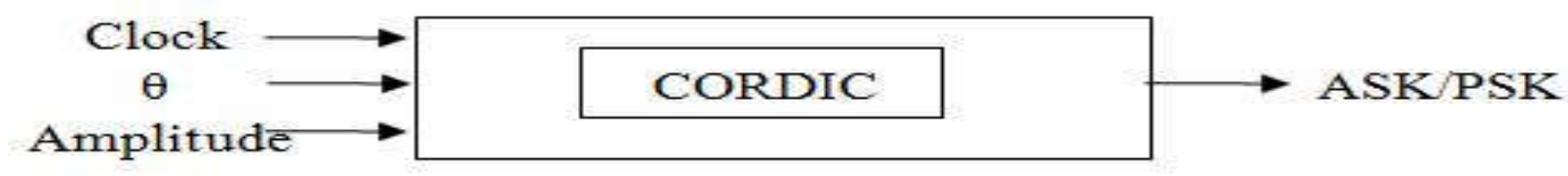

Fig.4.Digital modulation architecture

\section{RESULTSAND DISCUSSION}

Quartus II Altera is used as simulator and the waveforms were obtained from ModelSim-Altera before it was embedded into FPGA. The inputs and outputs were based on 16 bits' calculation with fixed point Q1.14. The CORDIC itself have restricted angle from $-\pi / 2$ to $\pi / 2$, but all other angle can actually be obtained using trigonometric properties. Fig. 4-6 show various inputs of angle within the first quadrant for the ASK modulation. From the Equations of the ASK, for amplitude 0, the output was 0. While for amplitude 1, the output was as in Equation (4). Table 3 shows the summary of the ASK modulation for the first quadrant after 10th iteration compared with the calculation value.

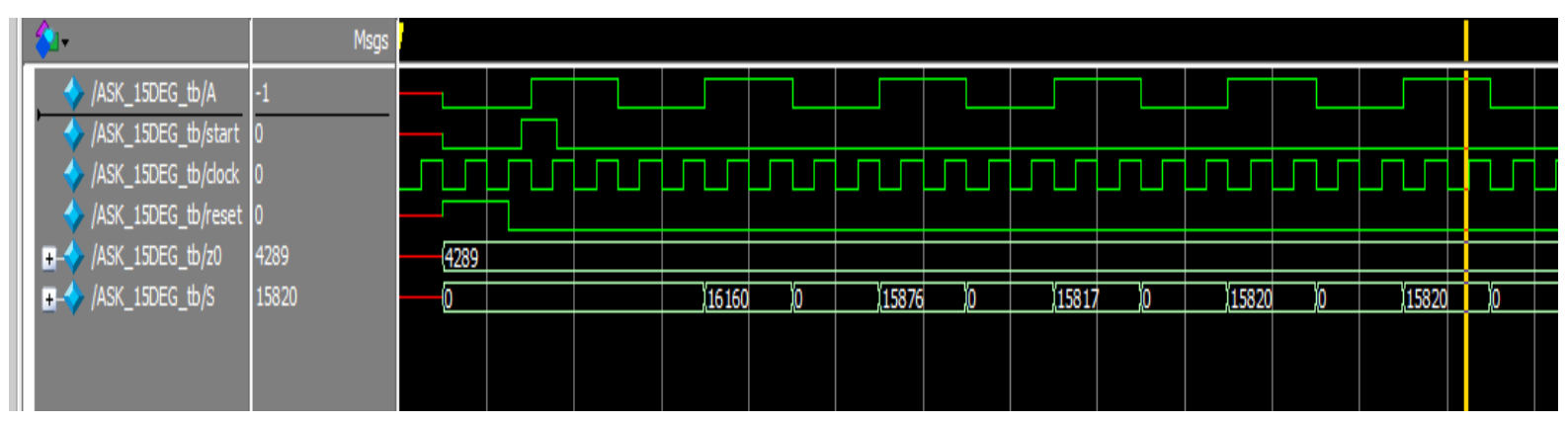

Fig.5.Simulation of 15 degree

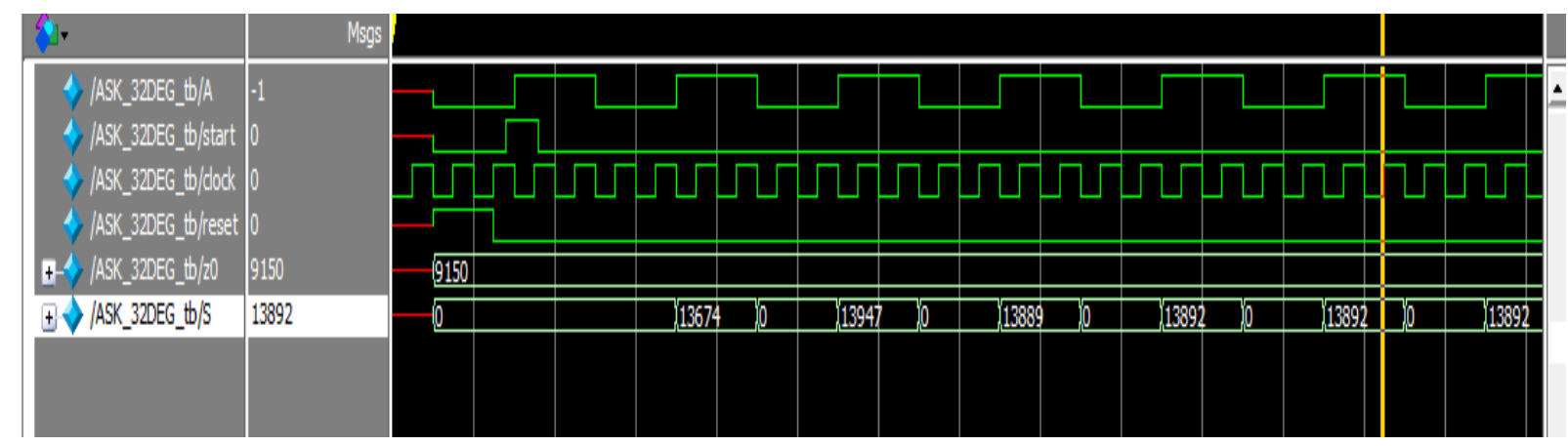

Fig.6.Simulation of 32 degree 


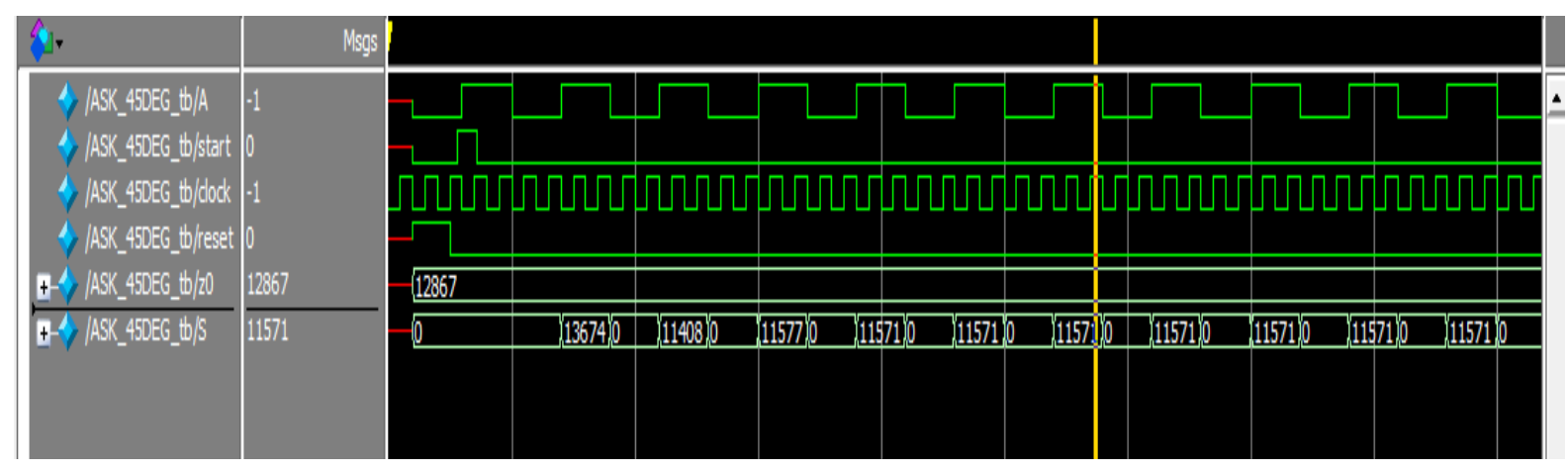

Fig.7.Simulation of 45 degree

Table 3. ASK modulation results after 10th iterations

\begin{tabular}{ccc}
\hline Angle & CalculatedResult, $\operatorname{Cos} \boldsymbol{\theta}$ & SimulatedResult, Cos $\boldsymbol{\theta}$ \\
\hline 15 & 15819 & 15820 \\
17 & 15661 & 15660 \\
30 & 14162 & 14187 \\
32 & 13897 & 13892 \\
45 & 11499 & 11571 \\
47 & 11181 & 11173 \\
60 & 8153 & 8182 \\
62 & 7667 & 7667 \\
75 & 4226 & 4238 \\
77 & 3676 & 3682 \\
\hline
\end{tabular}

Fig. 8-10 show the simulation results for PSK. Random angle was chosen to prove the algorithm's hypothesis. In the PSK modulation, when the amplitude is 0 , the output was negative of the real value as in Equation (7). 


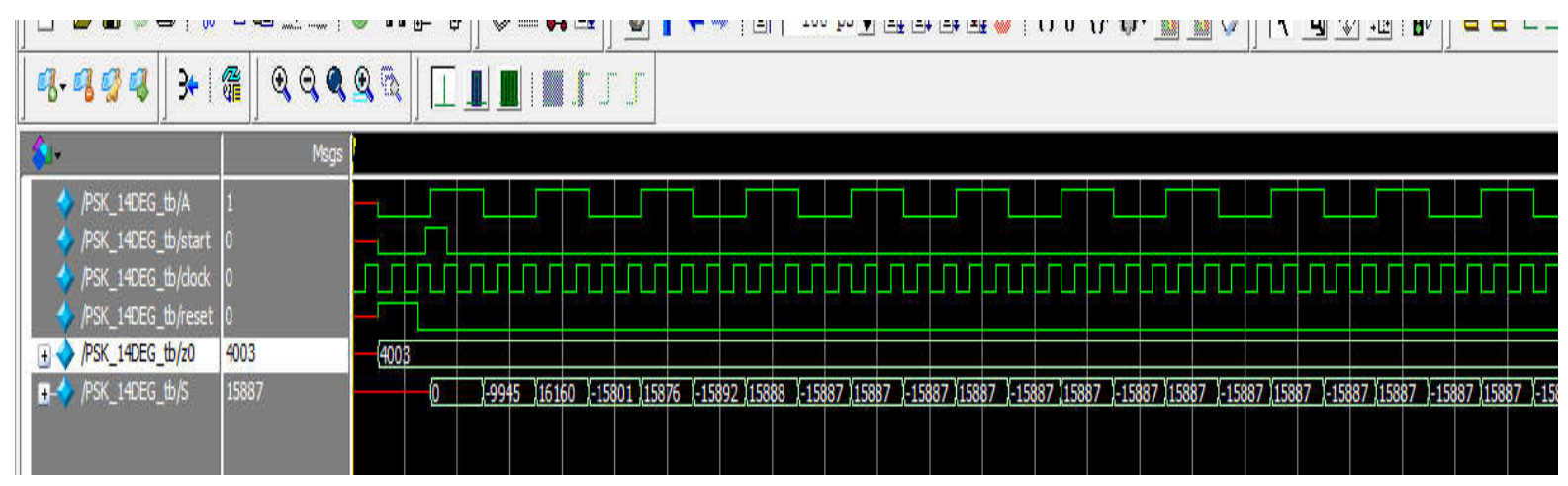

Fig.8.Simulation of 14 degree

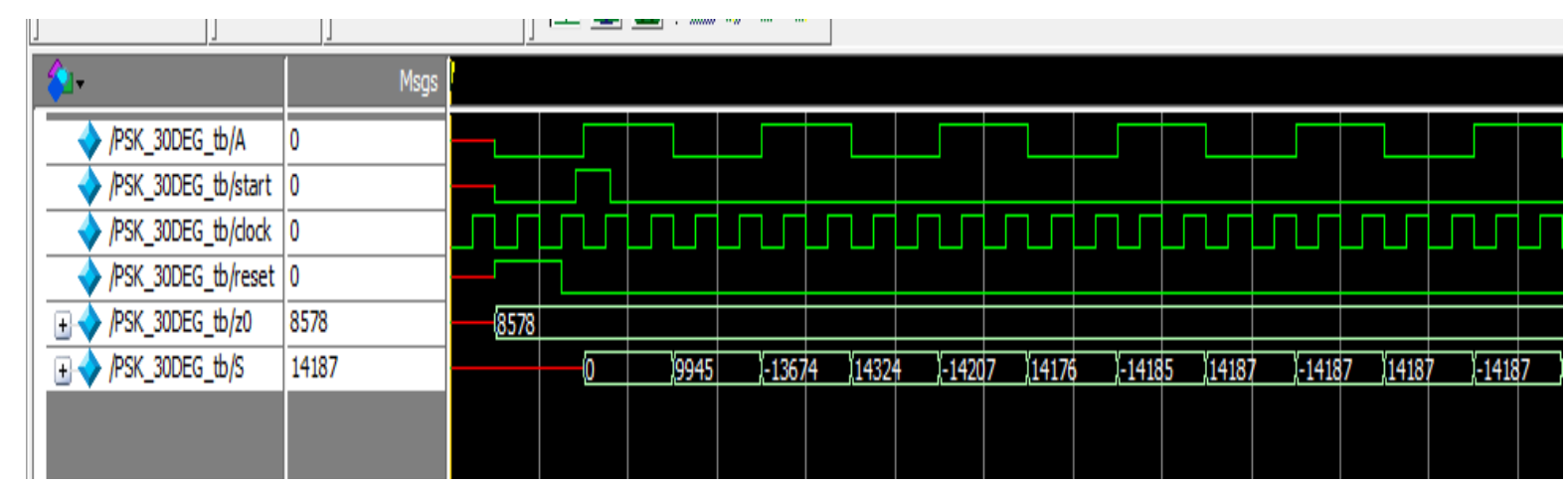

Fig.9.Simulation of 30 degree

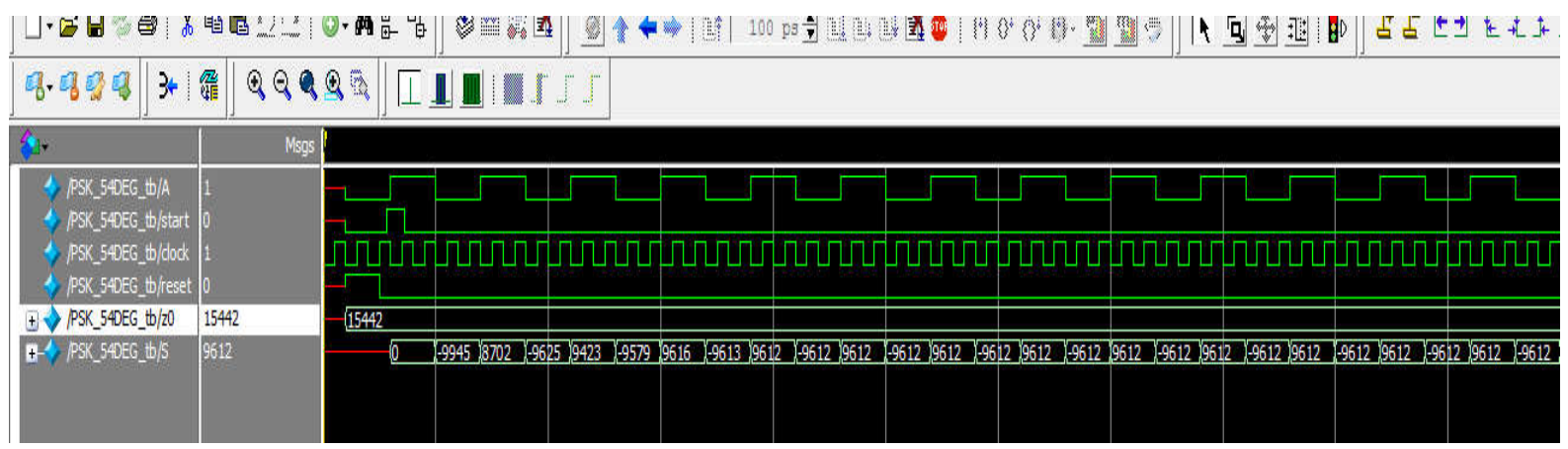

Fig.10.Simulation of 54 degree

Table 4. PSK modulation for 10th iteration

\begin{tabular}{ccccc}
\hline Degree & \multicolumn{2}{c}{ Calculated Result, Cos } & \multicolumn{2}{c}{ SimulatedResult, Cos } \\
& $\mathbf{A = 1}$ & $\mathbf{A = 0}$ & $\mathbf{A = 1}$ & $\mathbf{A = 0}$ \\
\hline 10 & 16124 & -16124 & 16125 & -16125 \\
14 & 15884 & -15884 & 15885 & -15885 \\
20 & 15380 & -15380 & 15402 & -15402 \\
24 & 15402 & -15402 & 14973 & -14973 \\
30 & 14193 & -14193 & 14192 & -14192 \\
\hline
\end{tabular}




\begin{tabular}{lllll}
\hline 34 & 13594 & -13594 & 13592 & -13592 \\
40 & 12559 & -12559 & 12549 & -12549 \\
44 & 11790 & -11790 & 11790 & -11790 \\
50 & 10511 & -10511 & 10512 & -10512 \\
54 & 9605 & -9605 & 9604 & -9604 \\
\hline
\end{tabular}

Table 4 shows an example of input and output required to operate in ASK modulation. Implementation of FPGA had been done and the output produced is shown as in Table 5.

Table 5. FPGA input and output for ASK modulation

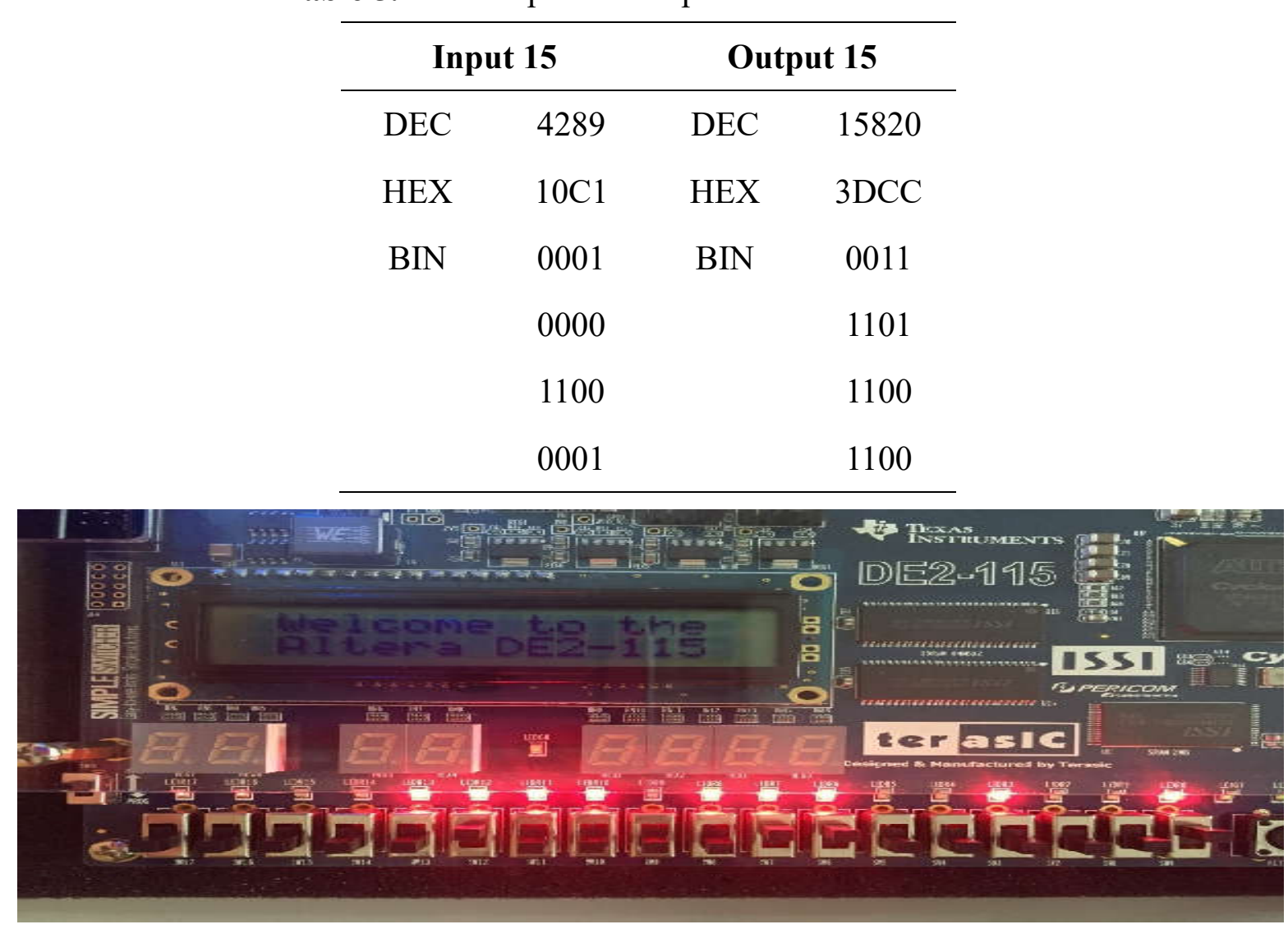

Fig.10. Implementation of FPGA for 15 degree

Table 6 shows an example of input and output required to operate for PSK modulation. Implementation of FPGA had been done and the output produced is shown as in Fig.11.Implementation of FPGA for 10 degree. 
Table 6. Input and output value for 10 degree

\begin{tabular}{ccc}
\hline Degree & Input & Output \\
& 10 & 10 \\
\hline DEC & 2859 & 16125 \\
HEX & $0 \mathrm{~B} 2 \mathrm{~B}$ & $3 \mathrm{EFD}$ \\
BIN & 0000101100101011 & 0011111011111101 \\
\hline
\end{tabular}

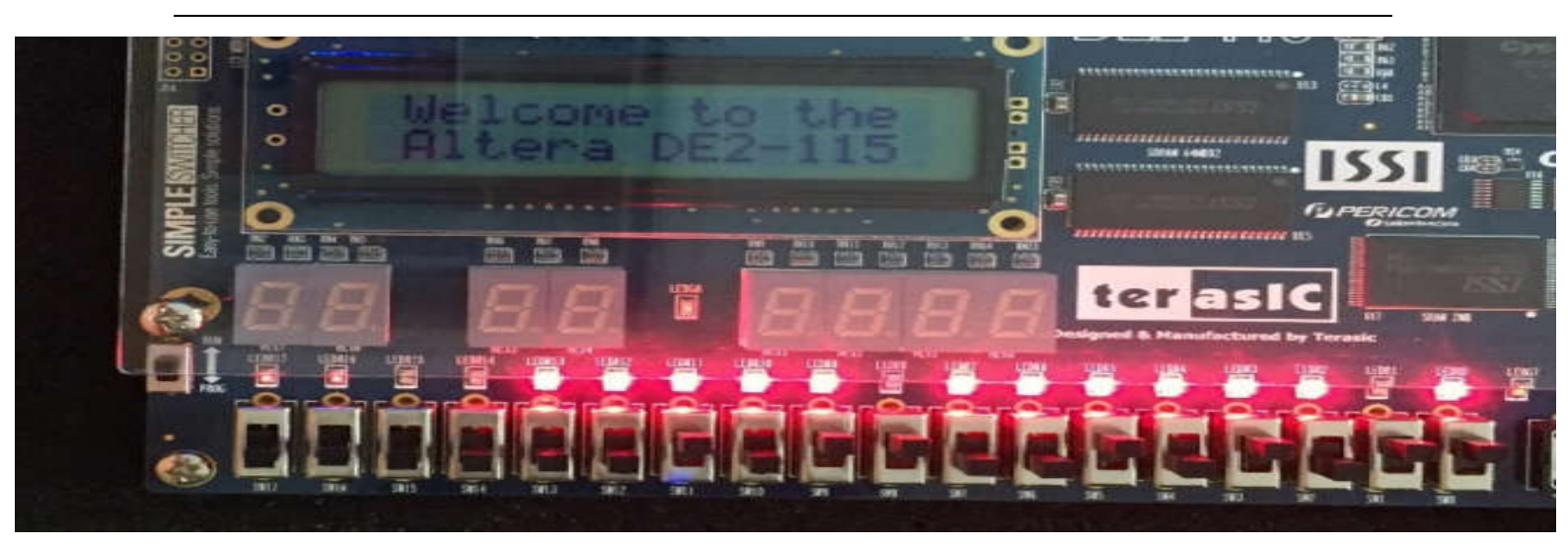

Fig.11.Implementation of FPGA for 10 degree

The advantage that CORDIC offers over other algorithms are that it does not required multiplication or division blocks instead it works only with shifter, adder/subtracted and a small look up table. This reduces the hardware requirement drastically and provides reasonably good speed and are ideal for FPGA implementation [5].

\section{CONCLUSION}

The CORDIC credited to Jack E. Volder is derived from the general rotation algorithm. The results obtained from the calculation of CORDIC algorithm and the simulation using Quartus II software were recorded and compared. It shows that CORDIC algorithm can be used to implement digital modulation with almost accurate results. It is found that the highest error of difference occur between the calculation and simulation value was only $0.006 \%$, which can be conclude that this algorithm is almost accurate and reliable for real time applications.

\section{ACKNOWLEDGEMENTS}

This research work is supported by the UPNM Short Grant (UPNM/2015/GPJP/2/TK/05). 


\section{REFERENCES}

[1] Agilent Technologies (AT).Agilent AN 1298: Digital modulation in communications systems-An Introduction: Application note.California: AT, 2005

[2] Jahgirdar R D. Study of digital modulation. Odisha: National Institute of Technology Rourkela, 2010

[3] Rajaram S, Gayathre R. FPGA implementation of digital modulation schemes. International Journal of Innovative Research in Science, Engineering and Technology, 2014, 3(3):1526-1531

[4] Jahagirdir R D. Study of digital modulation techniques. PhD thesis, Odisha: National Institute of Technology Rourkela, 2010

[5] Herbert D, Meyr H. CORDIC algorithms and architectures. Digital Signal Process Multimedia System, 1999, 2:623-655

[6] Timberlake C F. Anthocyanins in fruit and vegetables. In J. Friend, \& M. J. C. Rhodes (Eds.), Recent advances in the biochemistry of fruit and vegetables. New York: Academic Press, 1981, pp. 221-247

[7] NikDaud N G, Hashim F R, Mustapha M, Badruddin M S. Hybrid modified booth encoded algorithm-carry save adder fast multiplier. In 5th IEEE International Conference on Information and Communication Technology for the Muslim World, 2012, pp. 1-6

[8] Volder J E. The Cordic trigonometric computing technique. IRE Transaction Electronics Computer, 1959, 8:330-334

[9] Meher K P, Valls J, Tso-Bing, Sridharan K, Maharatna K. 50 years of CORDIC: Algorithms, architectures, and applications. IEEE Transactions on Circuits and Systems I: Regular Papers,2009, 56(9):1893-1907

[10] Godbole B B, Nikam R H, Pandit M D. A review of Cordic algorithm in DDS based communication systems.International Journal of Emerging Technology and Advanced Engineering, 2014, 4(2):911-915

[11] Tarniceriu A, Spiridon S, Iordanche B. An analysis on digital modulation techniques for software defined ratio applications. International Semiconductor Conference, 2007, pp. $571-574$ 
[12] Moreno L. Sensitivity of PSK modulation techniques to nonlinear distortions. IEEE Transactions on Communications, 1979, 27(5):806-812

\section{How to cite this article:}

Mokhtar A S N, Karim S A, Chew S P, Dardin S M F S M, Supian L S, Hashim F R. Fpga implementation of cordic algorithm in digital modulation. J. Fundam. Appl. Sci., 2017, 9(3S), 279-293. 